\title{
TachoSil use in abdominal surgery: a review
}

This article was published in the following Dove Press journal:

Journal of Blood Medicine

II March 20I I

Number of times this article has been viewed

\section{Adriana Toro \\ Maurizio Mannino \\ Giulio Reale \\ Isidoro Di Carlo}

Department of Surgical Sciences, Organ Transplantation, and Advanced Technologies, University of Catania, Cannizzaro Hospital, Catania, Italy
Correspondence: Isidoro Di Carlo Associate Professor of Surgery, Department of Surgical Sciences, Organ Transplantation, and Advanced Technologies, University of Catania, Cannizzaro Hospital via Messina 829,

95126 Catania, Italy

Tel +39095 7264863

Fax +39095 7263020

Email idicarlo@unict.it
Abstract: The success of any surgical procedure is based on adequate hemostasis. Many different biomaterial products can be used to achieve that aim. The products that can be used during surgery may be classified as topical hemostats, sealants, and adhesives. Hemostats can clot blood. Sealants can create sealing barriers. Adhesives bond tissue together. Collagen, gelatin, and cellulose are hemostat agents. TachoSil ${ }^{\circledR}$ is a development of TachoComb ${ }^{\circledR}$ and TachoComb ${ }^{\circledR}$ H. TachoComb is made with equine collagen, bovine thrombin, bovine aprotinin, and human fibrinogen. The clinical efficacy of TachoSil was shown firstly by a clinical study of hepatic surgery. In the study, TachoSil proved to be superior to argon beamer in obtaining effective and fast intraoperative hemostasis. Following the study, many applications in different fields of surgery have been reported in the literature. The use of TachoSil in open abdominal surgery and its relevant results have encouraged the use of TachoSil in laparoscopic surgery. Unfortunately, its use in laparoscopy has not become as popular as it is in open surgery, due to a lack of efficacious techniques. Immunologic reactions to compounds of TachoSil and the transmission of infectious diseases are two major risks concerning topical hemostasis. Even though the risk of severe immunologic reactions to bovine material is low, TachoSil has gradually replaced all bovine material with material of human origin and has therefore eliminated the associated risks of bovine material. TachoSil has a good satisfaction rate among surgeons and reduces both the operating time for patients and the time spent in intensive care units.

Keywords: TachoSil, abdominal surgery, hemostasis

\section{Introduction}

The success of any surgical procedure is based on adequate hemostasis. ${ }^{1}$ Ineffective local hemostasis is the major cause of bleeding during surgery, but many other causes may be involved. Major bleeding can be controlled by standard surgical techniques, such as stitches, ligatures, or clips. Chemical, thermal, and mechanical procedures can be used during surgery to achieve hemostasis, especially when diffuse hemorrhages occur. Surgical tools such as electrocautery, argon beamer, or laser are often useful, but sometimes these methods do not achieve satisfying results. Many different biomaterial products can be used to achieve that aim. ${ }^{2}$

\section{Hemostatic agents}

The products that can be used during surgery may be classified as topical hemostats, sealants, and adhesives. ${ }^{3}$ Hemostats can clot blood. Sealants can create sealing barriers. Adhesives bond tissue together. Collagen, gelatin, and cellulose are hemostat agents. 
Collagen is derived from bovine collagen. As with all bovine collagen products, it contains a small amount of bovine serum protein, so it should not be used in patients who are allergic to this product. Collagens exist as sponges of various sizes or in syringes. The most commonly used hemostatic collagens include FloSeal ${ }^{\circledR}$ (Baxter, Zurich, Switzerland), TissuFleece ${ }^{\circledR}$ (Baxter, Zurich, Switzerland), and GentaFleecel ${ }^{\circledR}$ (Baxter, Zurich, Switzerland). ${ }^{2}$ Gelatin sponges are a protein mixture obtained from collagen and Spongostan $^{\circledR}$ (Johnson \& Johnson, North Yorkshire, UK)/ Surgifoam ${ }^{\circledR}$ (Ethicon 360 Somerville, NJ, USA) and are a diffuse product. Oxidized celluloses are derived from cotton cellulose and can be provided in single or multiple sheets. In addition to the mechanical effect, the cellulosic acid within the product facilitates hemostasis by denaturating blood proteins. The most commonly commercial agent used in this group is Surgicel ${ }^{\circledR}$ (Ethicon 360 Somerville, NJ, USA). ${ }^{2}$ Fibrin glue is an example of a sealant composed of a fibrinogen concentrate and thrombin solutions. Thrombin solutions are currently divided into two categories: commercial products and those created in the laboratory. Tissucol $^{\circledR}$ (Baxter, Zurich, Switzerland), Tisseel ${ }^{\circledR}$ (Baxter, Zurich, Switzerland), Beriplast ${ }^{\circledR}$ (Nycomed Linz, Austria), $\mathrm{TachoSil}^{\circledR}$ (Nycomed Linz, Austria), and Quixil ${ }^{\circledR}$ (Omrix, Rhode-St-Genese, Belgium) are the most commonly used commercial products. ${ }^{4}$ The cyanoacrilates are a group of fast-acting adhesives that are currently approved for topical external use.

\section{Design, composition, and action of TachoSil}

TachoSil is a development of TachoComb ${ }^{\circledR}$ (Nycomed Linz, Austria) and TachoComb ${ }^{\circledR} \mathrm{H}$ (Nycomed Linz, Austria). TachoComb is made with equine collagen, bovine thrombin, bovine aprotinin, and human fibrinogen. TachoComb $\mathrm{H}$ contains human thrombin in place of bovine thrombin. TachoSil contains only human fibrinogen with equine collagen as a carrier. It does not contain aprotinin or any other component of bovine origin. TachoSil works by mimicking the final steps of the natural blood-clotting process, creating a fibrin clot at the surgical site to achieve hemostasis in 3-5 minutes. TachoSil consists of a white collagen sponge coated on one side with fibrinogen and thrombin. Riboflavin gives a yellow appearance to the coated side (Figure 1). One of the most important achievements of TachoSil compared with its precursors is the possibility of storage at room temperature, avoiding the necessity of refrigeration or freezing. The product is ready to use, which represents an advantage when it has to be used quickly.

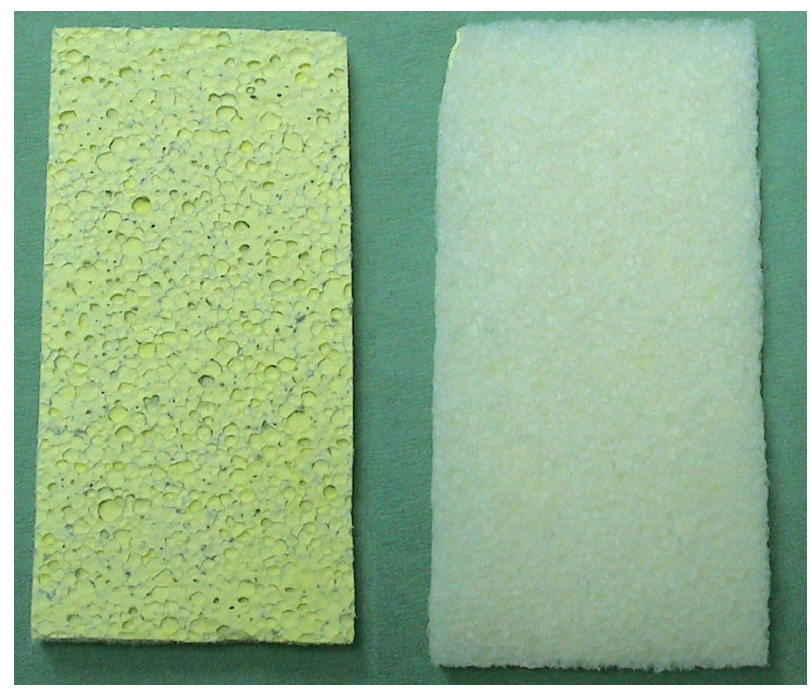

Figure I The yellow section represents the active part of TachoSil that has to be applied on to the liver surface.

TachoSil becomes active when the product comes into contact with bleeding or leaking tissue. The dry coating dissolves and releases the coagulation factors fibrinogen and thrombin and initiates the final steps in the blood coagulation process. During this process, thrombin converts fibrinogen into fibrin monomers, which spontaneously polymerize, and endogenous factor XIII catalyzes the crosslinking of fibrin, creating a firm, mechanically stable network. The resulting fibrin clot ensures effective hemostasis and sealing, thus preventing leaks. When the patch is in the appropriate place, the honeycomb-like matrix of the sponge folds in, resulting in air- and liquid-tight sealing of the tissue. TachoSil will be enzymatically degraded and safely absorbed by the body within 12 weeks of application.

TachoSil is indicated not only for hemostasis but also to provide tissue sealing, to support sutures, to prevent adhesions and erosions, to protect nerves, and to occlude structures such as bronchioles, lymph vessels. or bile ducts.

The sourcing of human coagulation factors of TachoSil is controlled and processed according to World Health Organization regulations, which provide a high degree of safety against human pathogens, including HIV and hepatitis $\mathrm{C}$ virus. Allergic reactions or cellular/humoral immunity following surgical implantation to purified collagen as formulated in TachoSil have never been detected. Equine collagen is a solid, rod-shaped molecule that is free of immunogenic epitopes. There are no registered reports on immunologic reactions to the equine collagen.

TachoSil is contraindicated in patients with hypersensitivity to any of the active substances or any of the excipients. 
It should be administered to pregnant or lactating women only if it is clearly necessary.

\section{Clinical application in abdominal surgery \\ Hepatic surgery}

The clinical efficacy of TachoSil was shown by a clinical study of hepatic surgery. ${ }^{5}$ It was the first prospective, randomized, open, controlled, multicentre trial with intraoperative as well as postoperative assessment of efficacy and a 1-month follow-up period of two groups of patients requiring secondary hemostasis during planned liver resection. The first group of patients was treated using carrier-bound fibrin sealant, and the second group was treated using argon beamer. The results showed a significant superiority of TachoSil over argon beamer regarding the time of hemostasis. In the study, TachoSil proved to be superior to argon beamer in obtaining effective and fast intraoperative hemostasis. After the study, many applications in different fields of surgery have been reported in the literature.

In the field of hepatic surgery, a second recent trial has been performed to demonstrate the efficacy and safety of TachoSil versus argon beamer in liver resection. ${ }^{6}$ The primary endpoint of the international, multicenter, randomized, controlled, surgical trial was the hemostasis time after starting the randomized intervention to obtain secondary hemostasis. Secondary endpoints were drainage duration, volume, and content. In the two groups of patients, the mean hemostasis time was less when TachoSil was used (3-6 minutes) compared with argon beamer (5.0 minutes $P=0.008)$. Postoperative drainage volume, drainage fluid, and drainage duration did not differ between the two groups. The study confirmed that TachoSil hemostasis is significantly faster than argon beamer after liver resection.

Another clinical trial supports the use of TachoSil. In the study, 115 patients were distributed into groups for major and minor hepatectomies, with or without application of a carrier-bound collagen sponge on the raw surface of the liver. Postoperative mortality, incidence and severity of postoperative surgical complications, and length of hospital stay were the main outcome measures. Postoperative drainage output volume, transfusion requirements, and changes in biochemical parameters were the secondary outcome measures. The fibrin sealant after major liver resection was effective in decreasing drainage volume, postoperative blood transfusion, complications, and hospital stay. ${ }^{7}$ To facilitate the decrease of drainage output volume, a "new Glisson surface" on resected liver should be made by a fibrin sealant. Therefore, all the liver surface should be sealed starting 1-2 $\mathrm{cm}$ above Glisson of the cut surface (Figure 2). ${ }^{8}$

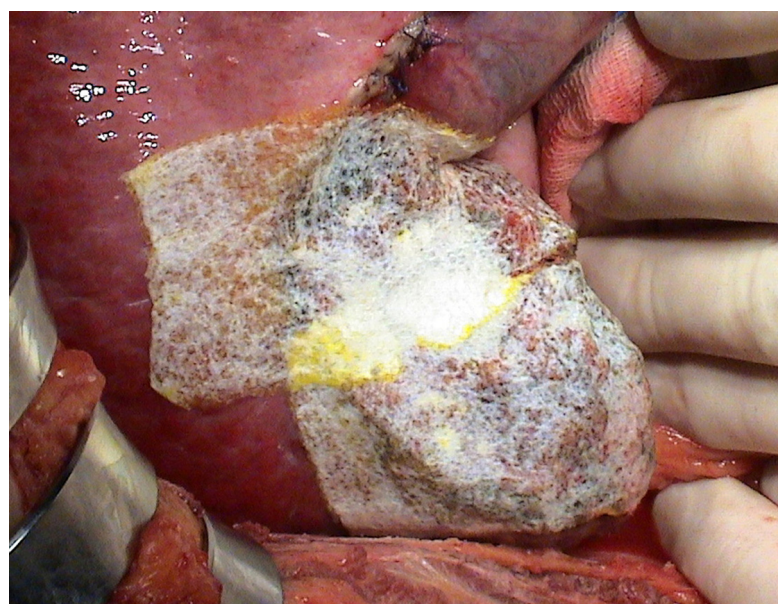

Figure $\mathbf{2}$ TachoSil correctly placed on the liver surface after hepatic resection.

Two experimental studies have focused on two different aspects of the product. In an experimental liver resection model in pigs by Erdogan et al, ${ }^{9}$ the application of a collagen patch on the resection surface was as effective as liquid fibrin sealants in obtaining hemostasis and sealing biliary ducts, but the adhesive strength of the sponge on the resection surface was superior. In particular, in this experimental study, the sealant increased pressure in the biliary system by infusing a saline solution with an automatic pump through a catheter in the common bile duct. This condition mimics the same effects that bile or blood has on the collagen sponge surface during the postoperative days, but it is condensed in a few minutes, and the pressure achieved inside the bile ducts is much higher than in clinical conditions (up to $120 \mathrm{~mm} \mathrm{Hg}$ ). The pressure in the biliary system was recorded and the resection surface was examined to identify leakage. The fibrinogen-coated collagen patch showed that the adhesive dressing always became loose, with the fleece material lifting off at the sealing level, but no bleeding or bile leakage was observed.

A second, more recent, experimental study investigated the efficacy of TachoSil in stopping severe bleeding in a coagulopathic pig model with blunt liver injury. ${ }^{10}$ The study analyzed two groups of pigs that were previously prepared by splenectomy and cystotomy and by inducing coagulopathy. Subsequently, a grade III liver injury was induced. At this point, animals were randomly assigned to receive a placebo patch or TachoSil. Coagulation parameters and hemodynamic variables as well as the chosen treatment were monitored for 2 hours after injury and patch placement. All animals treated with TachoSil survived, whereas $100 \%$ of the control group died before reaching the end of the observation period $(P<0.001)$.

A clinical report on liver transplantation has also been published. ${ }^{11}$ As bile leaks are a frequent complication of 
adults after liver transplantation, the authors compared surgical complications in patients who had the cut surface of the donor liver treated with a patch with those in whom the cut surface of the liver was treated with fibrin glue. The results of the study show that using a fibrinogen collagen sponge patch may reduce bile leaks from the cut surface of the liver during adult right lobe split liver transplantation.

Finally, in the field of hepatic surgery, TachoSil has also been used for life-threatening bleeding from a portal vein anastomosis during a cadaveric liver transplantation. ${ }^{12}$ A massive hemorrhage was seen in the area of the portal anastomosis after declamping, and control was achieved by application of the TachoSil patch around the portal vein. In this case, the role of the surgeons in applying slight finger pressure for several minutes was crucial. In fact, it is important to note that once the patch is in place, it should not be replaced or removed.

\section{Pancreatic surgery}

Many fields of abdominal surgery use this device because it is effective in hemostasis and also prevents leakage. Pancreatic surgery is one of the most difficult surgical fields due to the risk of pancreatic fistula. Two clinical studies concerning pancreatic surgery and the use of precursors of TachoSil have been reported in the literature. The first of these studies concerns patients submitted to surgery for acute pancreatitis, pancreas carcinoma, and chronic pancreatitis. ${ }^{13}$ The second study compares two groups of patients in whom the distal pancreatic stump after pancreatic resection has been treated using suture or stapler for closure with TachoComb in almost $50 \%$ of both groups. Both studies have small sample sizes, and no conclusive data are provided about the efficacy of the sealant agent in preventing pancreatic fistula.

Recently, a manuscript concerning the clinical application of TachoSil has been published. It concerns a group of patients submitted for pancreatic resection with pancreaticojejunal anastomosis protected by TachoSil. The results were not statistically significant, but they suggested possible advantages of TachoSil use in the prevention of postoperative pancreatic fistula. ${ }^{14}$

\section{Spleen}

Benefits of TachoSil application for spleen trauma ${ }^{15}$ or fragile or atraumatic damaged spleen in spleen management have been reported. ${ }^{16}$ In oncohematologic diseases, TachoSil may be useful in clotting and for blood composition disorders. ${ }^{17}$

\section{Gastrointestinal surgery}

Experimental and clinical studies on the sealing of colorectal anastomoses have previously been performed in order to reduce the rate of leakage, but with divergent results. However, comparatively fewer studies have been performed on anastomotic healing using a fibrin glue-coated patch. For this reason, an experimental basic scientific study in mice investigated the effect of fibrin glue-coated collagen patches on the healing process of colonic anastomoses in situations of adverse healing processes (technical deficiency and peritonitis).${ }^{18}$ Colonic anastomoses were carried out in 206 mice and randomized into six groups from complete anastomoses to sealed complete anastomoses in the presence of bacterial peritonitis. The evaluation of postoperative course data revealed the beneficial effect of additional sealing with TachoSil in high-risk experimental anastomotic healing. Sealing incomplete anastomoses resulted in significantly lower lethality and leakage rates. ${ }^{18}$

A second experimental study analyzed 11 pigs submitted to a double intestinal anastomosis treated with and without sealing with a collagen patch coated with fibrin glue. The pigs were observed for a time variable from 1 week to 6 weeks. The observation period was followed by in vivo examination under general anesthesia. Any difference concerning abdominal pathology, in vivo bursting pressure, or degree of stenosis was recorded. The collagen fleeces were in situ in all anastomoses. ${ }^{19}$

Surgery for peptic ulcer perforation is one of the most common emergency procedures carried out in the Western world; however, its mortality risk remains significantly high, despite improvement in surgical techniques, anesthesia, and critical care. Graham's patch omentoplasty represents the treatment of choice for both open and laparoscopic surgery. An experience of the gastric wall being repaired by a fullthickness suture followed by TachoSil patch application, which was finally covered by the classic omental patch, has been reported in the literature. ${ }^{20}$

\section{Inguinal hernia repair}

TachoSil has been investigated for the treatment of inguinal hernia repair. A recently published study examined what new absorbable materials can be used in the treatment of inguinal hernia with the same efficacy as the traditionally used polypropylene mesh. The authors performed an experimental study and a clinical study comparing direct hernia repair methods using Lichtenstein's operation in humans after implantation of polypropylene mesh or TachoSil for fascia transversalis reinforcement regarding postoperative 
pain and complications, time needed for patient mobilization, and recurrences. Patients implanted with TachoSil had significantly lower postoperative pain scores on a visual analog scale and used lower doses of analgesic. The overall incidence of postoperative complications was significantly reduced with the use of TachoSil. The incidence of recurrences after 24-month follow-up was the same in both groups. The study showed that TachoSil has the same short-term efficacy in hernia treatment as the standard polypropylene mesh, with a reduction in postoperative pain and analgesic use and a decrease in overall postoperative complications. ${ }^{21}$

\section{Laparoscopy}

The use of TachoSil in open abdominal surgery and its relevant results have encouraged the use of TachoSil in laparoscopic surgery. Unfortunately, its use in laparoscopy has not become as popular as it is in open surgery, due to the lack of efficacious techniques. Firstly, the passage of the patch via the laparoscopic cannula causes the exfoliation of the collagen compound due to its fragility. Not only does the product come into the abdominal cavity without its integrity but also when it comes into contact with blood or fluid, the fibrin-coated part is immediately activated and its manipulation becomes very difficult. The patch should be treated without touching any organ or fluid, and it should be applied accurately the first time. If this passage is made in the wrong way, the patch should not be used anymore, and another one should be inserted in the abdominal cavity. Moreover, when this patch is used to cover the liver surface after hepatic resection, pressure cannot be exercised uniformly as in open surgery, so a fluid collection can be found between the liver surface and the TachoSil in the postoperative period. This collection is usually asymptomatic, and no treatment, such as external drainage, is needed.

\section{Efficacy and safety}

Immunologic reactions to compounds of TachoSil and the transmission of infectious diseases are the two major risks concerning topical hemostasis. Differently from TachoComb and TachoComb $\mathrm{H}$ (both containing material of bovine origin), and even though the risk of severe immunologic reactions to bovine material is low, TachoSil has gradually replaced all bovine material with material of human origin and therefore has eliminated the associated risks of bovine material. The collagen is derived from horse tendons, which could potentially still cause immunological reactions, but collagens generally have low immunogenicity, so immunologic reactions to equine collagen are very unlikely. ${ }^{22}$
Another risk is the transmission of infectious diseases. There is a potential risk of transmission of micro-organisms from blood samples that are used to produce TachoSil, even if, according to the manufacturer, blood comes only from selected donors and all the active measures against enveloped viruses are used. Nonenveloped viruses are more resistant, especially the parvovirus B19. Infections with parvovirus B19 can lead to serious conditions with aplastic crisis. This is particularly dangerous for pregnant women, so the use of TachoSil is not recommended in pregnancy. Even if the risk exists, no reports of parvovirus transmission with TachoSil can be found in the literature. Limited data exist about the prions that may cause Creutzfeldt-Jakob disease. Although prions can be found in blood, there have been no reports of transmission by manufactured plasma products.

TachoSil has a good satisfaction rate among surgeons and reduces both the operating time for patients and time spent in intensive care units. ${ }^{23}$

\section{Disclosure}

The authors report no conflicts of interest in this work.

\section{References}

1. Spotnitz WD. Fibrin sealant: past, present, and future: a brief review. World J Surg. 2010;34:632-634.

2. Seyednejad H, Imani M, Jamieson T, Seifalian AM. Topical haemostatic agents. Br J Surg. 2008;95:1197-1225.

3. Spotnitz WD, Burks S. Hemostats, sealants, and adhesives: components of the surgical toolbox. Transfusion. 2008;48:1502-1516.

4. Dunn CJ, Goa KL. Fibrin sealant: a review of its use in surgery and endoscopy. Drugs. 1999;58:863-886.

5. Frilling A, Stavrou GA, Mischinger HJ, et al. Effectiveness of a new carrier-bound fibrin sealant versus argon beamer as haemostatic agent during liver resection: a randomised prospective trial. Langenbecks Arch Surg. 2005;390:114-120.

6. Fischer L, Seiler CM, Broelsch CE, et al. Hemostatic efficacy of TachoSil in liver resection compared with argon beam coagulator treatment: an open, randomized, prospective, multicenter, parallel-group trial. Surgery. 2011;149:48-55.

7. Briceño J, Naranjo A, Ciria R, et al. A prospective study of the efficacy of clinical application of a new carrier-bound fibrin sealant after liver resection. Arch Surg. 2010;145:482-488.

8. Di Carlo I, Toro A. Sealing all of the resection liver surface to maximize the adhesive strength of the carrier-bound fibrin sealant. Arch Surg. 2011;146:239.

9. Erdogan D, de Graaf W, van Gulik TM. Adhesive strength of fibrinogencoated collagen patch or liquid fibrin sealant in an experimental liver resection model in pigs. Eur Surg Res. 2008;41:298-302.

10. Grottke O, Braunschweig T, Daheim N, et al. Effect of TachoSil in a coagulopathic pig model with blunt liver injuries. J Surg Res. March 4 , 2010. [Epub ahead of print].

11. Toti L, Attia M, Manzia TM, et al. Reduction in bile leaks following adult split liver transplant using a fibrin-collagen sponge: a pilot study Dig Liver Dis. 2010;42:205-209.

12. Apestegui C, Breitenstein S, Dutkowski P, Clavien PA. Control of severe portal bleeding by carrier-bound fibrin sealant. Surg Today. 2009;39:363-365. 
13. Lorenz U, Maier M, Steger U, et al. Analysis of closure of the pancreatic remnant after distal pancreatic resection. HPB (Oxford). 2007;9: 302-307.

14. Chirletti P, Caronna R, Fanello G, et al. Pancreaticojejunostomy with application of fibrinogen/thrombin-coated collagen patch (TachoSil) in Roux-en-Y reconstruction after pancreaticoduodenectomy. J Gastrointest Surg. 2009;13:1396-1398.

15. Carbon RT, Baar S, Waldschmidt J, et al. Innovative minimally invasive pediatric surgery is of therapeutic value for splenic injury. $J$ Pediatr Surg. 2002;37:1146-1150.

16. Schwaitzberg SD, Chan MW, Cole DJ, et al. Comparison of poly-N-acetyl glucosamine with commercially available topical hemostats for achieving hemostasis in coagulopathic models of splenic hemorrhage. $J$ Trauma. 2004;57:S29-S32.

17. Tagliabue F, D'Angelo C, Zuccon W, et al. Use of TachoSil in splenectomy in patients with clotting and blood composition disorders. Minerva Chir. 2007;62:73-78.

18. Pantelis D, Beissel A, Kahl P, et al. The effect of sealing with a fixed combination of collagen matrix-bound coagulation factors on the healing of colonic anastomoses in experimental high-risk mice models. Langenbecks Arch Surg. 2010;395:1039-1048.
19. Nordentoft T, Rømer J, Sørensen M. Sealing of gastrointestinal anastomoses with a fibrin glue-coated collagen patch: a safety study. J Invest Surg. 2007;20:363-369.

20. Di Carlo I, Pulvirenti E, Toro A. Use of a fibrinogen- and thrombin-coated patch for peptic ulcer perforation repair. Hepatogastroenterology. 2009;56:575-577.

21. Arslani N, Patrlj L, Kopljar M, et al. Advantages of new materials in fascia transversalis reinforcement for inguinal hernia repair. Hernia. 2010;14:617-621.

22. Horowitz B, Busch M. Estimating the pathogen safety of manufactured human plasma products: application to fibrin sealants and to thrombin. Transfusion. 2008;48:1739-1753.

23. Anegg U, Rychlik R, Smolle-Jüttner F. Do the benefits of shorter hospital stay associated with the use of fleece-bound sealing outweigh the cost of the materials? Interact Cardiovasc Thorac Surg. 2008;7:292-296.
Journal of Blood Medicine

\section{Publish your work in this journal}

The Journal of Blood Medicine is an international, peer-reviewed, open access, online journal publishing laboratory, experimental and clinical aspects of all topics pertaining to blood based medicine including but not limited to: Transfusion Medicine; Blood collection, Donor issues, Transmittable diseases, and Blood banking logistics; Immunohematology; Artificial and alternative

\section{Dovepress}

blood based therapeutics; Hematology; Biotechnology/nanotechnology of blood related medicine; Legal aspects of blood medicine; Historical perspectives. The manuscript management system is completely online and includes a very quick and fair peer-review system. Visit http://www.dovepress.com/ testimonials.php to read real quotes from published authors. 